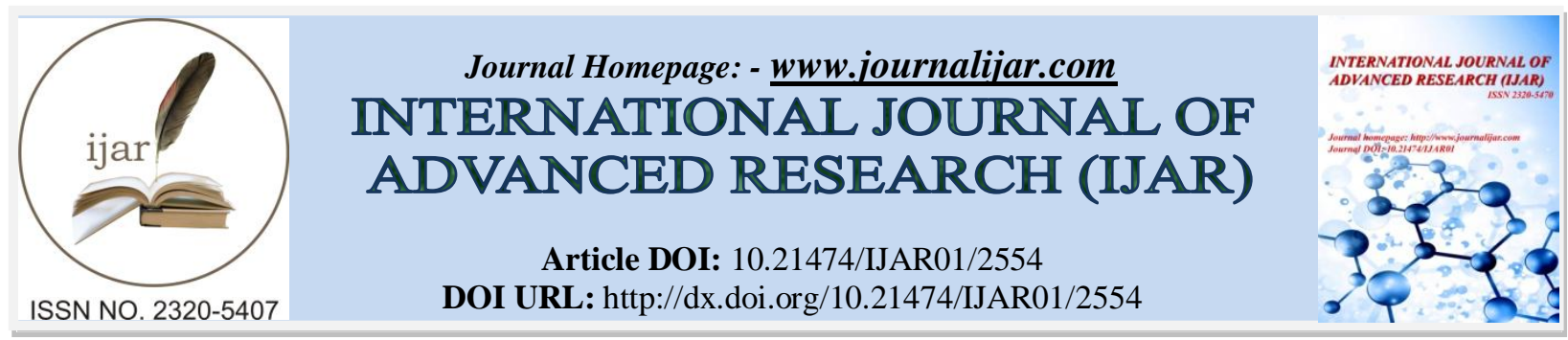

RESEARCH ARTICLE

\title{
ETHNOBOTANICAL SURVEY OF MEDICINAL PLANTS AGAINST SOME COMMON DISEASES IN ALIGARH DISTRICT, U.P., INDIA.
}

Yogendra Singh.

Department of Botany Divya Nand Vidyamandir Mahavidyalaya, Sandila, Hardoi.

\section{Manuscript Info}

\section{Manuscript History}

Received: 27 October 2016

Final Accepted: 25 November 2016

Published: December 2016

Key words:-

Aligarh district, Ethnobotany,

Phytoremedy,Traditional use.

\section{Abstract}

Since the ancient time, plant species have been used as the source of phytomedicine by the human beings. Plants are the richest source of medicine due to the presence of biochemical, which are useful to cure the various diseases. The present paper provides the information on the phytoremedies practiced by the Hakim, Vaidh and Local people of interior area of Aligarh district, U.P., India. During the floristic survey for collection the ethnomedicinal informations, 61 plant species belonging to 35 Families, 57 Genera were found to be used to cure various common diseases like amoebiosis, bronchitis, cold \& cough, diarrhoea, dysentery etc.

Copy Right, IJAR, 2016,. All rights reserved.

\section{Introduction:-}

From ancient times, the plants have been used as sources of medicines by the tribal or human being. The traditional medical knowledge of plants and their use by indigenous cultures are not only useful for conservation of cultural traditions, but also for community health care and drug development the present and future. The present floristic study deals with various common plant species having medicinal values in Aligarh district. Various kinds of ethnomedicinal plants like herbs, shrubs, weeds, tree etc. are used in different mode of administration in curing the different diseases. Geographically, Aligarh district lies $27^{\circ} 35^{\prime}$ to $28^{\circ} 11^{\prime}$ North latitude and $77^{\circ} 29^{\prime}$ to $78^{\circ} 38^{\prime}$ East longitude. It is bounded by Gautam Budh Nagar, Bulandshahr and Badaun on the north, whereas it is spread up to Haryana in the west. Topographically, it presents a shallow like trough appearance with high Ganga and Yamuna banks of extreme rim. River Yamuna flows on the north-west region of the district and Ganga separates the district from Badaun of the north-east end. All over the world, everybody is familiar to Aligarh district of U.P. due to presence of Aligarh Muslim University.

\section{Material and Methods:-}

Field trips were organized for collecting the ethnobotanical information of plants by using the interviews and questionnaires with practitioners and experienced men. While noting the information in the record file at the time of survey, every care is taken to record the local name, flowering \& fruiting time of plants and their parts and diseases name. The parts of the different plant species are different for the treatment of various common diseases. Plant species are identified by using relevant flora (Duthie, 1903-1929). The ethnomedicinal uses of the plants are compared with available Scientific Literatures (Katewa et al. 2001), (Kumar and Chauhan, 2005), (Murthy et al. 2013), (Pandey and Kumar, 2006), (Rahul, 2013), (Rani et al. 2009), (Sinhababu and Banerjee, 2013), (Sharma et al. 2010), (Yumnamcha et al. 2014).

Corresponding Author:- Yogendra Singh.

Address:- Department of Botany Divya Nand Vidyamandir Mahavidyalaya, Sandila, Hardoi. 


\section{Results:-}

While exploting the plants of Aligarh district during research from floristic and ethnobotanical point of view, author came across some ethnobotanical plant species, which were used to cure various common diseases. 61 ethnomedicinal plant species belonging to 35 families and 57 genera were enumerated in the present work. 26 plant species and 31 plant species were used for single drug treatments and multidrug treatment respectively. Data of medicinal plants clearly describes plant nature, local name, occurrence, flowering and fruiting time, plant part used and disease name in Table. Leaf constitute was the highest (19) of utilization followed by stem \& its parts (bark 12, rhizome 2, twig gum 1, stem 2 ), root (9), fruit (7), seed (7), whole plant (7), flower (5) and bulb (2).

Table :- List of Some Ethnomedicinal Plants of Aligarh district.

\begin{tabular}{|c|c|c|c|c|c|c|c|c|}
\hline $\begin{array}{l}\text { S. } \\
\text { no. }\end{array}$ & $\begin{array}{l}\text { Natu } \\
\text { re }\end{array}$ & Botanical name & Family & Local name & Occurrence & $\begin{array}{l}\text { Fls/Frts } \\
\text { Time }\end{array}$ & $\begin{array}{l}\text { Parts } \\
\text { used }\end{array}$ & Disease \\
\hline \multirow[t]{2}{*}{1.} & \multirow[t]{2}{*}{$\begin{array}{l}\text { Shru } \\
\mathrm{b}\end{array}$} & \multirow[t]{2}{*}{$\begin{array}{l}\text { Abrus } \\
\text { precatorius } \mathrm{L} .\end{array}$} & \multirow[t]{2}{*}{ Fabaceae } & \multirow[t]{2}{*}{ Ratti } & \multirow[t]{2}{*}{$\begin{array}{l}\text { Waste } \\
\text { place and } \\
\text { Road side }\end{array}$} & \multirow[t]{2}{*}{$\begin{array}{l}\text { Aug- } \\
\text { Sep/ } \\
\text { Sep-oct }\end{array}$} & Leaf & $\begin{array}{l}\text { Bronchitis, } \\
\text { Cold \& } \\
\text { Cough. }\end{array}$ \\
\hline & & & & & & & Root & $\begin{array}{l}\text { Cold \& } \\
\text { Cough. }\end{array}$ \\
\hline \multirow[t]{2}{*}{2.} & \multirow{2}{*}{$\begin{array}{l}\text { Shru } \\
\text { b }\end{array}$} & \multirow{2}{*}{$\begin{array}{l}\text { Abutilon indicum } \\
\text { L. }\end{array}$} & \multirow[t]{2}{*}{ Malvaceae } & \multirow[t]{2}{*}{ Kanghi } & \multirow{2}{*}{$\begin{array}{l}\text { Waste } \\
\text { place Road } \\
\text { side }\end{array}$} & \multirow{2}{*}{$\begin{array}{l}\text { Aug- } \\
\text { Jan/ } \\
\text { Jan-Mar }\end{array}$} & Leaf & Dysentery. \\
\hline & & & & & & & Root & Amoebiosis. \\
\hline 3. & Tree & $\begin{array}{l}\text { Acacia catechu } \\
\text { L. }\end{array}$ & Mimosaceae & Khairi & Road side & $\begin{array}{l}\text { Jul- } \\
\text { Aug/ } \\
\text { Sep- } \\
\text { Mar }\end{array}$ & Bark & Bronchitis. \\
\hline \multirow[t]{2}{*}{4.} & \multirow[t]{2}{*}{ Tree } & \multirow[t]{2}{*}{ Acacia nilotica $\mathrm{L}$} & \multirow[t]{2}{*}{ Mimosaceae } & \multirow[t]{2}{*}{ Babul } & \multirow[t]{2}{*}{ Road side } & \multirow[t]{2}{*}{$\begin{array}{l}\text { Jul-Oct/ } \\
\text { Nov- } \\
\text { Feb }\end{array}$} & Bark & $\begin{array}{l}\text { Cold, } \\
\text { Whooping } \\
\text { cough. }\end{array}$ \\
\hline & & & & & & & $\begin{array}{l}\text { Twig } \\
\text { Gum }\end{array}$ & Dysentery. \\
\hline \multirow[t]{2}{*}{5.} & \multirow[t]{2}{*}{ Herb } & \multirow[t]{2}{*}{$\begin{array}{l}\text { Achyranthes } \\
\text { aspera } \mathrm{L} .\end{array}$} & \multirow[t]{2}{*}{ Amaranthaceae } & \multirow[t]{2}{*}{ Chirchitta } & \multirow{2}{*}{$\begin{array}{l}\text { Semi dry } \\
\text { place, } \\
\text { Road side }\end{array}$} & \multirow{2}{*}{$\begin{array}{l}\text { Throug } \\
\text { h out } \\
\text { of year }\end{array}$} & Bark & $\begin{array}{l}\text { Blood } \\
\text { dysentery, }\end{array}$ \\
\hline & & & & & & & Seed & $\begin{array}{l}\text { Cold \& } \\
\text { Cough. }\end{array}$ \\
\hline \multirow[t]{2}{*}{6.} & \multirow{2}{*}{$\begin{array}{l}\text { Shru } \\
\mathrm{b}\end{array}$} & \multirow{2}{*}{$\begin{array}{l}\text { Adhatoda vasica } \\
\text { Nees. }\end{array}$} & \multirow[t]{2}{*}{ Acanthaceae } & \multirow[t]{2}{*}{ Piyabans } & \multirow[t]{2}{*}{ Garden } & Oct-Jan/ & Leaf & Bronchitis. \\
\hline & & & & & & Jan-Mar & Flower & Bronchitis. \\
\hline 7. & Tree & $\begin{array}{l}\text { Aegle marmelos } \\
\text { L. }\end{array}$ & Rutaceae & Bel & Road side & $\begin{array}{l}\text { Apr- } \\
\text { May/ } \\
\text { May- } \\
\text { July }\end{array}$ & Fruit & $\begin{array}{l}\text { Diarrhoea, } \\
\text { Dysentery. }\end{array}$ \\
\hline 8. & Tree & Ailanthus & Simaroubaceae & Ulloo & Planted in & Feb- & Bark & Amoebiosis. \\
\hline & & excelsa Roxb. & & & $\begin{array}{l}\text { garden \& } \\
\text { road side }\end{array}$ & $\begin{array}{l}\text { Apr/ } \\
\text { Apr- } \\
\text { June }\end{array}$ & Gum & $\begin{array}{l}\text { Diarrhoea, } \\
\text { Dysentery. }\end{array}$ \\
\hline 9. & Herb & Allium cepa $\mathrm{L}$. & Liliaceae & Piyaj & $\begin{array}{l}\text { Cultivated } \\
\text { Field }\end{array}$ & - & Bulb & $\begin{array}{l}\text { Cold \& } \\
\text { Cough. }\end{array}$ \\
\hline 10. & Herb & Allium sativum $\mathrm{L}$ & Liliaceae & Lahsun & $\begin{array}{l}\text { Cultivated } \\
\text { Field }\end{array}$ & - & Bulb & $\begin{array}{l}\text { Cold \& } \\
\text { Cough. }\end{array}$ \\
\hline 11. & Herb & Aloe vera Tourn. & Liliaceae & Ghinkwar & $\begin{array}{l}\text { Side of } \\
\text { fruit } \\
\text { orchards }\end{array}$ & $\begin{array}{l}\text { End } \\
\text { winter }\end{array}$ & Leaf & $\begin{array}{l}\text { Cold \& } \\
\text { Cough. }\end{array}$ \\
\hline 12. & Tree & $\begin{array}{l}\text { Alstonia } \\
\text { scholaris L. }\end{array}$ & Apocynaceae & Chitwan & Road side & $\begin{array}{l}\text { Jan- } \\
\text { Mar/ } \\
\text { May-Jul }\end{array}$ & Bark & $\begin{array}{l}\text { Diarrhoea, } \\
\text { Dysentery. }\end{array}$ \\
\hline 13. & Herb & $\begin{array}{l}\text { Amaranthus } \\
\text { spinosus } \mathrm{L} .\end{array}$ & Amaranthaceae & Kanta chouli & $\begin{array}{l}\text { Waste } \\
\text { place }\end{array}$ & Aug-Jan & Root & Amoebiosis. \\
\hline 14. & Herb & Argemone & Papaveraceae & Satyanasi & Waste and & Oct- & Whole & Whooping \\
\hline
\end{tabular}




\begin{tabular}{|c|c|c|c|c|c|c|c|c|}
\hline & & mexicana $\mathrm{L}$. & & & Dry place & $\begin{array}{l}\text { Apr/ } \\
\text { Apr- } \\
\text { May }\end{array}$ & & cough. \\
\hline 15. & Tree & $\begin{array}{l}\text { Artocarpus } \\
\text { integrifolia } \mathrm{L} .\end{array}$ & Moraceae & Katahal & $\begin{array}{l}\text { Cultivated } \\
\text { field }\end{array}$ & - & Fruit & $\begin{array}{l}\text { Diarrhoea, } \\
\text { Dysentery. }\end{array}$ \\
\hline 16. & Herb & $\begin{array}{l}\text { Bacopa monnieri } \\
\text { L. }\end{array}$ & Scrophuliaceae & Vermin & $\begin{array}{l}\text { Moist } \\
\text { place }\end{array}$ & $\begin{array}{l}\text { Aug- } \\
\text { Oct/ } \\
\text { Oct- } \\
\text { Nov } \\
\end{array}$ & Whole & Bronchitis. \\
\hline \multirow[t]{2}{*}{17.} & \multirow[t]{2}{*}{ Tree } & \multirow{2}{*}{$\begin{array}{l}\text { Bauhinia } \\
\text { purpurea } \mathrm{L} .\end{array}$} & \multirow[t]{2}{*}{ Caesalpinoidae } & \multirow[t]{2}{*}{ Kachnar } & \multirow{2}{*}{$\begin{array}{l}\text { Planted in } \\
\text { garden \& } \\
\text { roadsides }\end{array}$} & \multirow{2}{*}{$\begin{array}{l}\text { Nov- } \\
\text { Fab/ } \\
\text { Mar- } \\
\text { Apr } \\
\end{array}$} & Bark & Diarrhoea. \\
\hline & & & & & & & Root & $\begin{array}{l}\text { Cold \& } \\
\text { Cough. }\end{array}$ \\
\hline 18. & Tree & $\begin{array}{l}\text { Butea } \\
\text { monosperma } \\
\text { Lam. }\end{array}$ & Fabaceae & Dhak & $\begin{array}{l}\text { Rare but } \\
\text { road side }\end{array}$ & $\begin{array}{l}\text { Mar- } \\
\text { May }\end{array}$ & Gum & Dysentery. \\
\hline 19. & $\begin{array}{l}\text { Und } \\
\text { er } \\
\text { shru } \\
\text { b } \\
\end{array}$ & $\begin{array}{l}\text { Carissa } \\
\text { spinarum L. }\end{array}$ & Apocynaceae & Karaunda & $\begin{array}{l}\text { Side of } \\
\text { fruit } \\
\text { orchards }\end{array}$ & $\begin{array}{l}\text { Nov- } \\
\text { Dec/ } \\
\text { Dec- } \\
\text { Feb }\end{array}$ & Leaf & $\begin{array}{l}\text { Whooping } \\
\text { cough. }\end{array}$ \\
\hline 20. & Herb & $\begin{array}{l}\text { Centella asiatica } \\
\text { L. }\end{array}$ & Apiaceae & Brahmi & $\begin{array}{l}\text { Moist } \\
\text { place }\end{array}$ & $\begin{array}{l}\text { Feb- } \\
\text { Apr/ } \\
\text { Apr- } \\
\text { May } \\
\end{array}$ & Whole & $\begin{array}{l}\text { Diarrhoea, } \\
\text { Dysentery. }\end{array}$ \\
\hline 21. & Herb & $\begin{array}{l}\text { Corchorus } \\
\text { capsularis L. }\end{array}$ & Tiliaceae & Kharenti & $\begin{array}{l}\text { Cultivated } \\
\text { field }\end{array}$ & $\begin{array}{l}\text { Aug- } \\
\text { Oct }\end{array}$ & Leaf & Dysentery. \\
\hline 22. & $\begin{array}{l}\text { Und } \\
\text { er } \\
\text { shru } \\
\text { b } \\
\end{array}$ & $\begin{array}{l}\text { Coriandrum } \\
\text { sativum } \mathrm{L} .\end{array}$ & Apiaceae & Dhania & $\begin{array}{l}\text { Cultivated } \\
\text { field }\end{array}$ & Jan-Mar & Seed & $\begin{array}{l}\text { Amoebiosis, } \\
\text { Diarrhoea, } \\
\text { Dysentery. }\end{array}$ \\
\hline 23. & Herb & $\begin{array}{l}\text { Curcuma longa } \\
\mathrm{L},\end{array}$ & Zingiberaceae & Haldi & $\begin{array}{l}\text { Cultivated } \\
\text { field }\end{array}$ & Sep-Oct & $\begin{array}{l}\text { Rhizom } \\
\text { e }\end{array}$ & $\begin{array}{l}\text { Cold \& } \\
\text { Cough, } \\
\text { Dysentery. }\end{array}$ \\
\hline 24. & Herb & $\begin{array}{l}\text { Cynodon } \\
\text { dactylon (L.) } \\
\text { Pers. }\end{array}$ & Poaceae & Doob ghass & Waste field & \begin{tabular}{|l|} 
Most \\
parts of \\
year
\end{tabular} & Whole & $\begin{array}{l}\text { Diarrhoea, } \\
\text { Dysentery. }\end{array}$ \\
\hline 25. & Herb & $\begin{array}{l}\text { Cyperus } \\
\text { rotundus } \mathrm{L} .\end{array}$ & Cyperaceae & Motha & $\begin{array}{l}\text { Moist } \\
\text { place }\end{array}$ & $\begin{array}{ll}\text { Jul } & - \\
\text { Dec }\end{array}$ & Root & Dysentery. \\
\hline 26. & Herb & Datura metel L. & Solanaceae & Dhatura & Road side & $\begin{array}{l}\text { Dec- } \\
\text { Feb }\end{array}$ & Leaf & $\begin{array}{l}\text { Amoebiosis, } \\
\text { Diarrhoea. }\end{array}$ \\
\hline 27. & Herb & $\begin{array}{l}\text { Eclipta prostrata } \\
\text { L. }\end{array}$ & Asteraceae & $\begin{array}{l}\text { Kala } \\
\text { Bhangra }\end{array}$ & $\begin{array}{l}\text { Moist } \\
\text { place }\end{array}$ & \begin{tabular}{|l|} 
Most \\
part of \\
year
\end{tabular} & Leaf & $\begin{array}{l}\text { Bronchitis, } \\
\text { Cough, } \\
\text { Dysentery. }\end{array}$ \\
\hline 28. & Tree & $\begin{array}{l}\text { Eucalyptus } \\
\text { glabulus Labill. }\end{array}$ & Myrtaceae & Safeda & $\begin{array}{l}\text { Side of } \\
\text { orchards \& } \\
\text { road }\end{array}$ & Apr-Jun & Leaf & Bronchitis. \\
\hline 29. & Herb & $\begin{array}{l}\text { Euphorbia } \\
\text { prostrata Sims. }\end{array}$ & Euphorbiaceae & Gonemchi & $\begin{array}{l}\text { Waste } \\
\text { place }\end{array}$ & \begin{tabular}{|l|} 
Most \\
part of \\
year
\end{tabular} & Whole & Diarrhoea. \\
\hline 30. & Herb & $\begin{array}{l}\text { Evolvulus } \\
\text { alsinoides } \mathrm{L} .\end{array}$ & Convolvulaceae & Vishnukranta & $\begin{array}{l}\text { Road side, } \\
\text { in village }\end{array}$ & $\begin{array}{l}\text { Aug- } \\
\text { Oct/ } \\
\text { Oct- } \\
\text { Nov }\end{array}$ & Leaf & $\begin{array}{l}\text { Bronchitis, } \\
\text { Cold \& } \\
\text { Cough. }\end{array}$ \\
\hline 31. & Tree & $\begin{array}{l}\text { Ficus } \\
\text { benghalensis L. }\end{array}$ & Moraceae & Bargad & $\begin{array}{l}\text { Road side, } \\
\text { in villages }\end{array}$ & Mar-Jun & Bark & $\begin{array}{l}\text { Diarrhoea, } \\
\text { Dysentery. }\end{array}$ \\
\hline
\end{tabular}




\begin{tabular}{|c|c|c|c|c|c|c|c|c|}
\hline 32. & Tree & $\begin{array}{l}\text { Ficus racemosa } \\
\text { L. }\end{array}$ & Moraceae & Gular & $\begin{array}{l}\text { Road side, } \\
\text { in villages }\end{array}$ & Mar-Jun & Bark & $\begin{array}{l}\text { Diarrhoea, } \\
\text { Dysentery. }\end{array}$ \\
\hline 33. & Tree & $\begin{array}{l}\text { Ficus religiosa } \\
\text { L. }\end{array}$ & Moraceae & Pipal & $\begin{array}{l}\text { Road side, } \\
\text { in villages }\end{array}$ & $\begin{array}{l}\text { Mar- } \\
\text { May }\end{array}$ & Bark & $\begin{array}{l}\text { Diarrhoea, } \\
\text { Whooping } \\
\text { cough. }\end{array}$ \\
\hline 34. & Herb & $\begin{array}{l}\text { Leucas } \\
\text { cephalotes Roth. }\end{array}$ & Lamiaceae & Goma & & $\begin{array}{l}\text { Oct- } \\
\text { Nov/ } \\
\text { Nov- } \\
\text { Dec }\end{array}$ & Flower & $\begin{array}{l}\text { Cold \& } \\
\text { Cough. }\end{array}$ \\
\hline 35. & Herb & $\begin{array}{l}\text { Linum } \\
\text { usitatissimum } \mathrm{L} \text {. }\end{array}$ & Linaceae & Alsi & $\begin{array}{l}\text { Cultivated } \\
\text { field }\end{array}$ & $\begin{array}{l}\text { Jan- } \\
\text { Feb/ } \\
\text { Feb- } \\
\text { Mar } \\
\end{array}$ & Seed & $\begin{array}{l}\text { Cold \& } \\
\text { Cough. }\end{array}$ \\
\hline 36. & Tree & $\begin{array}{l}\text { Mangifera } \\
\text { indica } \mathrm{L} .\end{array}$ & Anacardiaceae & Aam & $\begin{array}{l}\text { Cultivated } \\
\text { in orchards }\end{array}$ & $\begin{array}{l}\text { Feb- } \\
\text { Apr/ } \\
\text { May-Jul }\end{array}$ & Bark & $\begin{array}{l}\text { Bleeding } \\
\text { dysentery. }\end{array}$ \\
\hline 37. & Herb & $\begin{array}{l}\text { Mentha } \\
\text { longifolia } \mathrm{L} .\end{array}$ & Lamiaceae & Podina & $\begin{array}{l}\text { Moist } \\
\text { place }\end{array}$ & $\begin{array}{l}\text { Oct- } \\
\text { Nov/ } \\
\text { Nov- } \\
\text { Dec }\end{array}$ & Leaf & $\begin{array}{l}\text { Diarrhoea, } \\
\text { Dysentery. }\end{array}$ \\
\hline 38. & Herb & $\begin{array}{l}\text { Mimusops elengi } \\
\text { L. }\end{array}$ & Sapotaceae & Maulsari & & $\begin{array}{l}\text { Apr- } \\
\text { May/ } \\
\text { May- } \\
\text { Jun }\end{array}$ & Fruit & Dysentery, \\
\hline 39. & Herb & $\begin{array}{l}\text { Momordica } \\
\text { charantia L. }\end{array}$ & Cucurbitaceae & Karela & $\begin{array}{l}\text { Cultivated } \\
\text { in orchards }\end{array}$ & $\begin{array}{l}\text { Jul-Sep/ } \\
\text { Sep-Oct }\end{array}$ & Leaf & $\begin{array}{l}\text { Diarrhoea, } \\
\text { Dysentery. }\end{array}$ \\
\hline \multirow[t]{2}{*}{40.} & \multirow[t]{2}{*}{ Herb } & \multirow[t]{2}{*}{$\begin{array}{l}\text { Musa } \\
\text { paradisiaca } \mathrm{L} \text {. }\end{array}$} & \multirow[t]{2}{*}{ Musaceae } & \multirow[t]{2}{*}{ Kela } & \multirow[t]{2}{*}{$\begin{array}{l}\text { Side of } \\
\text { orchards }\end{array}$} & \multirow[t]{2}{*}{-} & Fruit & $\begin{array}{l}\text { Diarrhoea, } \\
\text { Dysentery. }\end{array}$ \\
\hline & & & & & & & Rachis & Dysentery. \\
\hline \multirow[t]{2}{*}{41.} & \multirow[t]{2}{*}{ Herb } & \multirow[t]{2}{*}{$\begin{array}{l}\text { Ocimum } \\
\text { basilicum } \mathrm{L} .\end{array}$} & \multirow[t]{2}{*}{ Lamiaceae } & \multirow[t]{2}{*}{ Vantulsi } & \multirow[t]{2}{*}{$\begin{array}{l}\text { Waste } \\
\text { place }\end{array}$} & \multirow[t]{2}{*}{$\begin{array}{l}\text { Nov- } \\
\text { Dec/ } \\
\text { Dec-Jan }\end{array}$} & Leaf & $\begin{array}{l}\text { Dysentery, } \\
\text { Whooping } \\
\text { cough. }\end{array}$ \\
\hline & & & & & & & Flower & $\begin{array}{l}\text { Cold \& } \\
\text { Cough. }\end{array}$ \\
\hline 42. & Herb & $\begin{array}{l}\text { Oxalis } \\
\text { corniculata } \mathrm{L} .\end{array}$ & Oxalidaceae & Khati mithi & $\begin{array}{l}\text { Moist } \\
\text { place \& in } \\
\text { garden }\end{array}$ & $\begin{array}{l}\text { Dec- } \\
\text { Feb/ } \\
\text { Feb-Apr }\end{array}$ & Whole & $\begin{array}{l}\text { Diarrhoea, } \\
\text { Dysentery. }\end{array}$ \\
\hline 43. & Tree & $\begin{array}{l}\text { Pongamia } \\
\text { pinnata (L.) } \\
\text { Pierra. }\end{array}$ & Fabaceae & Papri & $\begin{array}{l}\text { Road side, } \\
\text { rare in } \\
\text { garden }\end{array}$ & $\begin{array}{l}\text { Mar- } \\
\text { May/ } \\
\text { May- } \\
\text { Jun }\end{array}$ & Seed & $\begin{array}{l}\text { Whooping } \\
\text { cough. }\end{array}$ \\
\hline \multirow[t]{2}{*}{44.} & \multirow[t]{2}{*}{ Tree } & \multirow{2}{*}{$\begin{array}{l}\text { Psidium guajava } \\
\text { L. }\end{array}$} & \multirow[t]{2}{*}{ Myrtaceae } & \multirow[t]{2}{*}{ Amroud } & \multirow{2}{*}{$\begin{array}{l}\text { Cultivated } \\
\text { in orchards }\end{array}$} & \multirow[t]{2}{*}{ May-Jul } & Stem & Amoebiosis. \\
\hline & & & & & & & Fruit & $\begin{array}{l}\text { Diarrhoea, } \\
\text { Dysentery. }\end{array}$ \\
\hline 45. & $\begin{array}{l}\text { Und } \\
\text { er- } \\
\text { shru } \\
\text { b }\end{array}$ & $\begin{array}{l}\text { Rauvolfia } \\
\text { serpentina (L.) } \\
\text { Benth. }\end{array}$ & Apocynaceae & Chotachand & $\begin{array}{l}\text { Rare, in } \\
\text { shady } \\
\text { place }\end{array}$ & $\begin{array}{l}\text { Mar- } \\
\text { Apr/ } \\
\text { Apr- } \\
\text { May } \\
\end{array}$ & Root & Diarrhoea. \\
\hline 46. & Herb & $\begin{array}{l}\text { Rungia repens } \\
\text { (L.) Nees. }\end{array}$ & Acanthaceae & Manga & $\begin{array}{l}\text { Moist, } \\
\text { shady } \\
\text { place }\end{array}$ & Oct- Jan & Whole & Cough. \\
\hline 47. & $\begin{array}{l}\text { Gras } \\
\mathrm{s}\end{array}$ & $\begin{array}{l}\text { Saccharum } \\
\text { officinalis L. }\end{array}$ & Poaceae & Ganna & $\begin{array}{l}\text { Cultivation } \\
\text { in field }\end{array}$ & $\begin{array}{ll}--- \\
\end{array}$ & Stem & $\begin{array}{l}\text { Constipation, } \\
\text { Diarrhoea. }\end{array}$ \\
\hline 48. & Tree & $\begin{array}{l}\text { Salvadora } \\
\text { persica } \mathrm{L} .\end{array}$ & Salvadoraceae & Pilu & $\begin{array}{l}\text { Garden, } \\
\text { road side }\end{array}$ & $\begin{array}{l}\text { Nov- } \\
\text { Feb/ }\end{array}$ & Leaf & $\begin{array}{l}\text { Cough, } \\
\text { Bronchitis. }\end{array}$ \\
\hline
\end{tabular}




\begin{tabular}{|c|c|c|c|c|c|c|c|c|}
\hline & & & & & & $\begin{array}{l}\text { Feb- } \\
\text { Mar }\end{array}$ & & \\
\hline 49. & $\begin{array}{l}\text { Und } \\
\text { er } \\
\text { shru } \\
\text { b }\end{array}$ & $\begin{array}{l}\text { Sida cordifolia } \\
\text { L. }\end{array}$ & Malvaceae & khirainti & $\begin{array}{l}\text { Dry place, } \\
\& \text { road } \\
\text { side }\end{array}$ & $\begin{array}{l}\text { Sep- } \\
\text { Dec }\end{array}$ & Leaf & Dysentery. \\
\hline 50. & Herb & $\begin{array}{l}\text { Sisymbrium irio } \\
\text { L. }\end{array}$ & Brassicaceae & Khumbkalan & $\begin{array}{l}\text { Moist } \\
\text { field, road } \\
\text { side }\end{array}$ & $\begin{array}{l}\text { Mar- } \\
\text { Apr }\end{array}$ & Seed & Diarrhoea. \\
\hline \multirow[t]{3}{*}{51.} & \multirow[t]{3}{*}{ Herb } & \multirow{3}{*}{$\begin{array}{l}\text { Solanum } \\
\text { xanthocarpum } \\
\text { Schrad. }\end{array}$} & \multirow[t]{3}{*}{ Solanaceae } & \multirow[t]{3}{*}{ Kateri } & \multirow{3}{*}{$\begin{array}{l}\text { Dry and } \\
\text { waste } \\
\text { place }\end{array}$} & \multirow{3}{*}{$\begin{array}{l}\text { May- } \\
\text { Aug/ } \\
\text { Aug- } \\
\text { Sep }\end{array}$} & Flower & $\begin{array}{l}\text { Whooping } \\
\text { cough. }\end{array}$ \\
\hline & & & & & & & Fruit & $\begin{array}{l}\text { Cold \& } \\
\text { Cough. }\end{array}$ \\
\hline & & & & & & & Root & $\begin{array}{l}\text { Cold \& } \\
\text { Cough. }\end{array}$ \\
\hline \multirow[t]{2}{*}{52.} & \multirow[t]{2}{*}{ Tree } & \multirow[t]{2}{*}{ Syzygium cumini } & \multirow[t]{2}{*}{ Myrtaceae } & \multirow[t]{2}{*}{ Jamun } & \multirow{2}{*}{$\begin{array}{l}\text { Side of } \\
\text { orchards }\end{array}$} & \multirow{2}{*}{$\begin{array}{l}\text { Apr- } \\
\text { Jun/ } \\
\text { Jun-Jul }\end{array}$} & Leaf & Diarrhoea. \\
\hline & & & & & & & Bark & $\begin{array}{l}\text { Diabetes, } \\
\text { Dysentery. }\end{array}$ \\
\hline \multirow[t]{3}{*}{53.} & \multirow[t]{3}{*}{ Tree } & \multirow{3}{*}{$\begin{array}{l}\text { Tamarindus } \\
\text { indica } \mathrm{L} \text {. }\end{array}$} & \multirow[t]{3}{*}{ Caesalpiniaceae } & \multirow[t]{3}{*}{ Imli } & \multirow{3}{*}{$\begin{array}{l}\text { Road side } \\
\text { and in } \\
\text { villages }\end{array}$} & \multirow{3}{*}{$\begin{array}{l}\text { Jul-Sep/ } \\
\text { Sep- } \\
\text { Nov }\end{array}$} & Leaf & Dysentery. \\
\hline & & & & & & & Flower & $\begin{array}{l}\text { Blood } \\
\text { dysentery. }\end{array}$ \\
\hline & & & & & & & Seed & $\begin{array}{l}\text { Cold \& } \\
\text { Cough. }\end{array}$ \\
\hline 54. & Tree & $\begin{array}{l}\text { Terminalia } \\
\text { arjuna Roxb. }\end{array}$ & Combretaceae & Arjun & Road side & $\begin{array}{l}\text { May- } \\
\text { Jun/ } \\
\text { Jul-Aug }\end{array}$ & Bark & Bronchitis. \\
\hline 55. & Herb & $\begin{array}{l}\text { Tribulus } \\
\text { terrestris } \mathrm{L} .\end{array}$ & Zygophyllaceae & Gokhru & $\begin{array}{l}\text { Dry and } \\
\text { waste } \\
\text { place }\end{array}$ & $\begin{array}{l}\text { Jul-Sep/ } \\
\text { Sep- } \\
\text { Nov }\end{array}$ & Fruit & Bronchitis. \\
\hline 56. & Herb & $\begin{array}{l}\text { Trigonella } \\
\text { foenum-graecum } \\
\text { L. }\end{array}$ & Fabaceae & Methi & $\begin{array}{l}\text { Cultivation } \\
\text { in field }\end{array}$ & $\begin{array}{l}\text { Jan- } \\
\text { Feb/ } \\
\text { Feb- } \\
\text { Mar } \\
\end{array}$ & Seed & Dysentery. \\
\hline 57. & $\begin{array}{l}\text { Und } \\
\text { er } \\
\text { shru } \\
\text { b }\end{array}$ & $\begin{array}{l}\text { Triumfetta } \\
\text { rhomboidea } \\
\text { Jacq. }\end{array}$ & Tiliaceae & Kasni & $\begin{array}{l}\text { Waste } \\
\text { place }\end{array}$ & $\begin{array}{l}\text { Aug- } \\
\text { Sep/ } \\
\text { Sep-Oct }\end{array}$ & Root & Bronchitis. \\
\hline 58. & Herb & $\begin{array}{l}\text { Vernonia } \\
\text { cinerea } \mathrm{L} .\end{array}$ & Asteraceae & Phulni & Road side & $\begin{array}{l}\text { Sep- } \\
\text { Feb/ } \\
\text { Feb- } \\
\text { Mar }\end{array}$ & Root & $\begin{array}{l}\text { Cold \& } \\
\text { Cough, } \\
\text { Diarrhoea. }\end{array}$ \\
\hline 59. & Herb & Vinca rosea L. & Apocynaceae & Sadawahar & $\begin{array}{l}\text { Cultivation } \\
\text { in field }\end{array}$ & $\begin{array}{l}\text { Most } \\
\text { part } \\
\text { of year }\end{array}$ & Leaf & $\begin{array}{l}\text { Blood } \\
\text { dysentery. }\end{array}$ \\
\hline 60. & Herb & $\begin{array}{l}\text { Zingiber } \\
\text { officinale L. }\end{array}$ & Zingiberaceae & Adarak & $\begin{array}{l}\text { Cultivation } \\
\text { in field }\end{array}$ & - & $\begin{array}{l}\text { Rhizom } \\
\mathrm{e}\end{array}$ & $\begin{array}{l}\text { Cold \& } \\
\text { Cough. }\end{array}$ \\
\hline 61. & $\begin{array}{l}\text { Shru } \\
\text { b }\end{array}$ & $\begin{array}{l}\text { Zyzyphus } \\
\text { nummularia } \\
\text { Burn f. }\end{array}$ & Rhamnaceae & Jhar Ber & $\begin{array}{l}\text { Side of } \\
\text { raiway } \\
\text { lines }\end{array}$ & $\begin{array}{l}\text { Jul-Sep/ } \\
\text { Sep- } \\
\text { Dec }\end{array}$ & Leaf & $\begin{array}{l}\text { Cold \& } \\
\text { Cough. }\end{array}$ \\
\hline
\end{tabular}

\section{Discussions:-}

The present paper provides the traditional and medicinal uses of plant species. These plant species have been used by practitioners and local experience people in the anterior area of Aligarh district. Most of the plant species are herbaceous nature. These plant species are used as phytoremedies to cure the various common diseases like 
amoebiosis, bronchitis, Cold \& Cough, diarrhoea, dysentery etc. Different plant parts such as bark, flower, fruit, leaf, roots, seeds, stem etc. are used for the treatment of diseases.

\section{Conclusion:-}

The present study is very helpful to list out various ethnomedicinal plants of Aligarh district. The paper provided here can be utilized to further studies on conservation and cultivation of ethnomedicinal plants, because most of the plant species are on the way of extinct due to pollution. The youth should also be encouraged to learn the traditional medicinal knowledge to preserve it from being lost with the older generation. The present paper briefly provides the ethnomedicinal information's related to 61 medicinal plant species.

\section{Acknowledgement:-}

The author is thankful to the practitioners and other experienced old men. I would like to thank my best friend Dr. Akshya kumar yadav (Ph.D. Scholar in AMU, Aligarh) for suggesting the problem and for incessant help and exquisite guidance. Their tireless efforts, immense patience, motivation and lively discussion throughout the course of present investigation, made working with them an enjoyable experience.

\section{References:-}

1. Duthie, J. F. (1903-1929). "Flora of the upper gangetic plain of the adjacent Siwalik and Sub-Himalayan tracts". Botanical Survey of India, Calcutta. Vol. 3.

2. Katewa, S. S., Chaudhary, B. L., Jain, A. and Takhar, H. K. (2001). "Some plants in folk medicine of Rajsamamd district (Rajasthan)". Ethnobotany.Vol. 13 (1 - 2): 129 - 134.

3. Kumar, S. and Chauhan, A. K. S. (2005). "Medicinal plants used by local inhabitants in Bharatpur district, Rajasthan". Ethnobotany. Vol. 17 (1 - 2): 179 - 183.

4. Murthy, S. M. S. and Vidyasagar, G. M. (2013). "Traditional knowledge on medicinal plants used in the treatment of respiratory disorders in Bellary district, Karnataka, India". Indian Journal of Natural products and Resources. Vol. 4 (2): 189 - 193.

5. Pandey, R.S. and Kumar, R.A. (2006). "An ethnobotanical study in the Vindhyan Region, Uttar Pradesh". Indian J. For. Vol. 29 (4): 389 - 394.

6. Rahul, J. (2013). "An Ethnobotanical study of Medicinal Plants in Taindol Village, District Jhansi, Region of Bundelkhand, Uttar Pradesh, India”. Journal of Medicinal Plants Studies. Vol. 1 (5): 59 - 71.

7. Rani, R., Gautam, R. and Gautam, R. K. (2009). "Floristic survey of medicinal plants in Sur Sarovar wet land, Kheetham, Agra, India". Journal of Applied and Natural Science. Vol. 1 (2): 196 - 200.

8. Sinhababu. A. and Banerjee, A. (2013). "Ethno-botanical Study of Medicinal Plants Used by Tribals of Bankura Districts, West Bengal, India”. Journal of Medicinal Plants Studies. Vol. 1 (3): 98 - 104.

9. Sharma, J., Painuli, R. M. and Gaur, R. D. (2010). "Plants used by the rural communities of district Shahjahanpur, Uttar Pradesh". Indian Journal of Traditional Knowledge. Vol. 9 (4): 798 - 803.

10. Yumnamcha, T., Nongthomba, U. and Devi, D. M. (2014). "Phytochemical screening and evaluation of genotoxicity and acute toxicity of aqueous extract of Croton tiglium L.". International Journal of Scientific and Research Publications, Vol. 4 (1): 436 - 439. 